Revista Tecné, Episteme y Didaxis: TED. Año 2014, Número

Extraordinario. ISSN Impreso: 0121-3814, ISSN web: 2323-0126

Memorias, Sexto Congreso Internacional sobre Formación de

Profesores de Ciencias. 08 al 10 de octubre de 2014, Bogotá

\title{
Actitudes hacia las ciencias de los estudiantes de NM1 del Colegio San Sebastián de Los Andes de la red educaUC
}

Romero, Israel ${ }^{1}$

Categoría 2. Trabajo de investigación (en proceso)

\section{Resumen}

El presente estudio tiene como objetivo identificar las actitudes hacia las ciencias de los estudiantes de NM1 mediante la aplicación del Test of Science Related Attitudes (TOSRA), el cual estandariza mediante subescalas las actitudes relacionadas con la ciencia, tales como implicaciones sociales de la ciencia, estilos de vida de los científicos, entre otros. Con la aplicación durante un semestre de un taller de ciencias, basado en la experimentación de temas asociados al currículum actual, los estudiantes expresan un cambio en sus actitudes. Para validar estos cambios, se aplicará un Post-test del TOSRA, el cual puede ser analizado estadísticamente aplicando McNemar, correlación de Pearson y la prueba T, comparando simultáneamente las actitudes según el sexo de la muestra.

\section{Palabras claves}

Actitudes hacia la ciencia, TOSRA, diferencia por sexo, taller de ciencias.

\section{Objetivos}

Identificar las actitudes hacia las ciencias de los estudiantes de NM1 mediante la aplicación del test de TOSRA.

Comparar según sexo las actitudes hacia las ciencias de los estudiantes de NM1.

Insertar en el plan de estudio un programa de ciencias experimental para determinar un cambio en las actitudes hacia las ciencias de los estudiantes de NM1.

'Colegio San Sebastián de Los Andes educaUC. iromero@educauc.cl 
Revista Tecné, Episteme y Didaxis: TED. Año 2014, Número Extraordinario. ISSN Impreso: 0121-3814, ISSN web: 2323-0126

Memorias, Sexto Congreso Internacional sobre Formación de Profesores de Ciencias. 08 al 10 de octubre de 2014, Bogotá

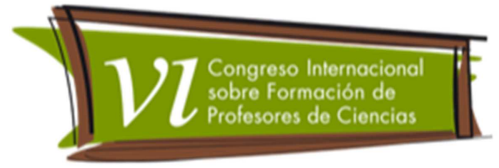

\section{Marco teórico}

En Chile, la enseñanza de las ciencias ha tenido distintos cambios curriculares que han generado un descontento social y una desmotivación por parte de los estudiantes para su estudio.

En el Marco Curricular de la Educación para el sector de ciencias se plantea que a futuro la marginalidad estará ligada a la carencia de conocimientos básicos en ciencias y a las formas de pensamiento propias a la investigación científica. Es por ello que resulta significativo estudiar las actitudes hacia la ciencia de los estudiantes, debido a las repercusiones que tiene el aprendizaje de las disciplinas científicas en las decisiones que toman los estudiantes en el futuro y en la percepción de los alcances de la ciencia y la tecnología en materias globales.

Las actitudes hacia la ciencia están relacionadas con el componente afectivo de cada individuo, haciendo referencia a la valoración de las personas hacia diversos objetos actitudinales, tales como, la ciencia escolar, los científicos, la preferencia por una carrera afín con ciencia, implicancias sociales asociadas con las ciencias, entre otros.

Al analizar las actitudes hacia la ciencia, investigaciones exponen la influencia de ciertas variables, como lo es el sexo de los estudiantes. En Chile, los resultados de la prueba PISA 2006 se observaron diferencias entre hombres y mujeres en el aprendizaje en ciencias, existiendo una diferencia significativa a favor de los hombres (448 puntos versus 426 puntos mujeres) manteniéndose en el año 2009. Estas diferencias en los desempeños en el área de las ciencias tiene consecuencias en la motivación para aprender la disciplina y en la actitud hacia el aprendizaje de ella en la escuela, lo que finalmente afecta los caminos que seguirán los estudiantes en el ámbito educacional y profesional.

\section{Metodología}

\section{Muestra}

La muestra constó de 65 alumnos (32 mujeres y 33 hombres) de primer año medio del Colegio San Sebastián de Los Andes perteneciente a la red educaUC. La selección de este nivel se basa en la desmotivación existente al estudio del taller de ciencias, el cual fue modificado curricularmente en el 2014, con el fin de obtener logros significativos en el aprendizaje y mayor apreciación hacia el estudio de las ciencias. Además, como la educación en Chile es generalizada durante los ocho primeros años de escolaridad, cuando alcanzan el noveno año 
Revista Tecné, Episteme y Didaxis: TED. Año 2014, Número Extraordinario. ISSN Impreso: 0121-3814, ISSN web: 2323-0126 Memorias, Sexto Congreso Internacional sobre Formación de Profesores de Ciencias. 08 al 10 de octubre de 2014, Bogotá

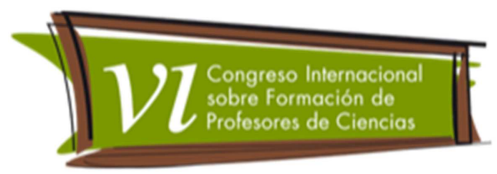

(primero medio), las ciencias naturales se dividen en Química, Física y Biología, donde los estudiantes no visualizan la integración de estas tres áreas en la revisión curricular de sus contenidos. La edad promedio de los estudiantes es de 14 años con un mínimo de 13 años y un máximo de 16 años. Los estudiantes pertenecen a dos cursos del nivel de estudio (Primero medio A y Primero medio B). Como la investigación está en proceso, después de la aplicación del pretest de TOSRA, se trabajará en el taller de ciencias dos horas semanales en el laboratorio de ciencias, según horario de trabajo de cada curso, hasta fines del primer semestre del año en curso.

\section{Instrumentos}

\section{-Escala de actitudes hacia la ciencia}

Se utilizó el Test of Science Related Attitudes (TOSRA, "Escala de actitudes relacionadas con la ciencia"). Esta escala, orientada a estudiantes de educación secundaria, está compuesta por 70 ítems, distribuidos en las siguientes subescalas: Implicaciones sociales de la ciencia; Estilo de vida de los científicos; Actitud hacia la investigación científica; Adopción de actitudes científicas; Agrado por las clases de ciencia; Interés por la ciencia durante el tiempo libre; Interés en carreras científicas.

Cada subescala se compone de 10 afirmaciones frente a las cuales los estudiantes deben señalar su agrado de acuerdo o desacuerdo en una escala de tipo Likert de cinco puntos. Las subescalas de TOSRA presentan una confiabilidad que oscila entre 0,67 y 0,93, y la confiabilidad global es de 0,90. La aplicación aplicación fue conjunta el 18 de marzo de 2014 y tuvo una duración de 45 minutos, aproximadamente.

\section{-Taller de ciencias}

El taller de ciencias permite aumentar el interés de los estudiantes en el área de las ciencias, el conocimiento de conceptos y procedimientos científicos, y la adquisición de nuevas competencias. Por consiguiente, las experimentaciones deben ser entendidas como herramientas metodológicas que permiten mejorar el aprendizaje científico escolar.

Desde esta perspectiva, el programa que se hará incorporará dispone de una selección e incorporación de actividades de experimentación que permiten promover la expresión de las propias ideas de los estudiantes sobre un 
Revista Tecné, Episteme y Didaxis: TED. Año 2014, Número Extraordinario. ISSN Impreso: 0121-3814, ISSN web: 2323-0126 Memorias, Sexto Congreso Internacional sobre Formación de Profesores de Ciencias. 08 al 10 de octubre de 2014, Bogotá

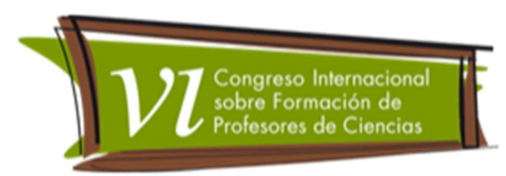

determinado fenómeno y el planteamiento de preguntas significativas que faciliten el aprendizaje y la construcción del conocimiento científico, acompañadas con sus planificaciones por semana de trabajo pedagógico.

\section{-Análisis de datos}

Se realizó un pretest del TOSRA con el fin de determinar inicialmente las actitudes de los estudiantes frente a las ciencias. Inicialmente, los análisis estadísticos son de tipo descriptivo e inferencial. Se utilizó la Prueba † para analizar la comparación de las actitudes de los estudiantes según sexo con un nivel de significancia del $5 \%$ $(p<0,05)$.

\section{Resultados}

Del total de subescalas del test, el que presentó la media más alta frente a una actitud positiva es la Adopción de actitudes científicas (A) (media = 18,54; SD = 2,44 ) y la subescala que presentó la media más alta frente a una actitud negativa fue las Implicaciones sociales de la ciencia (S) (media $=17,62$; SD $=2,68$ ). Por consiguiente, la subescala que presentó la media más baja frente a una actitud positiva fue el Interés en carreras científicas $(C)$ (media $=13,00 ; S D=3,77$ ) y la subescala que presentó la media más baja frente a una actitud negativa fue el Interés por la ciencia en el tiempo libre $(L)$ (media = 14,00; SD = 3,94) (Tabla 1).

\begin{tabular}{|c|c|c|c|c|c|c|c|}
\hline & $\begin{array}{l}\frac{\sigma}{\overline{0}} \\
\frac{\Phi}{\Sigma}\end{array}$ & 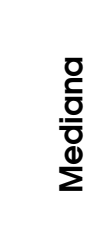 & $\begin{array}{l}\frac{0}{0} \\
\frac{0}{2}\end{array}$ & 品 & 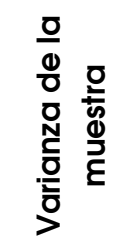 & $\begin{array}{l}\frac{n}{0} \\
0 \\
\frac{0}{5} \\
0\end{array}$ & 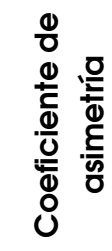 \\
\hline$S(+)$ & 17,95 & 18,00 & 18,00 & 2,45 & 5,98 & $-0,20$ & $-0,32$ \\
\hline$S(-)$ & 17,62 & 18,00 & 18,00 & 2,68 & 7,21 & $-0,04$ & $-0,14$ \\
\hline$N(+)$ & 17,49 & 17,00 & 21,00 & 3,38 & 11,44 & $-0,86$ & $-0,11$ \\
\hline$N(-)$ & 15,03 & 15,00 & 15,00 & 2,16 & 4,69 & 4,57 & 0,81 \\
\hline$I(+)$ & 17,66 & 18,00 & 18,00 & 3,37 & 11,38 & $-0,72$ & $-0,18$ \\
\hline I (-) & 15,35 & 15,00 & 13,00 & 2,68 & 7,20 & $-0,12$ & 0,01 \\
\hline
\end{tabular}


Revista Tecné, Episteme y Didaxis: TED. Año 2014, Número Extraordinario. ISSN Impreso: 0121-3814, ISSN web: 2323-0126 Memorias, Sexto Congreso Internacional sobre Formación de Profesores de Ciencias. 08 al 10 de octubre de 2014, Bogotá

\begin{tabular}{|l|c|c|c|c|c|c|c|}
$\mathbf{A}(+)$ & 18,54 & 19,00 & 20,00 & 2,44 & 5,94 & $-0,20$ & $-0,74$ \\
\hline $\mathbf{A ~ ( - )}$ & 17,51 & 18,00 & 18,00 & 2,67 & 7,13 & 0,80 & $-0,56$ \\
\hline $\mathbf{E ~ ( + )}$ & 14,66 & 15,00 & 14,00 & 3,90 & 15,23 & $-0,67$ & 0,21 \\
\hline $\mathbf{E ~ ( - )}$ & 16,98 & 17,00 & 15,00 & 2,87 & 8,27 & $-0,08$ & 0,14 \\
\hline $\mathbf{L ~ ( + )}$ & 13,49 & 13,00 & 13,00 & 4,48 & 20,07 & 0,13 & $-0,30$ \\
\hline $\mathbf{L}(-)$ & 14,00 & 14,00 & 16,00 & 3,94 & 15,50 & $-0,27$ & $-0,03$ \\
\hline $\mathbf{C ~ ( + )}$ & 13,00 & 13,00 & 13,00 & 3,77 & 14,19 & $-0,08$ & 0,27 \\
\hline $\mathbf{C ~ ( - )}$ & 14,55 & 14,00 & 12,00 & 3,28 & 10,75 & $-0,06$ & 0,28 \\
\hline
\end{tabular}

Analizando las diferencias de las medias en función del sexo de la muestra completa, se observa que no hay diferencias significativas en las actitudes frente a las ciencias entre los hombres y las mujeres ( $t=0,34, p>0,05$ ) (tabla 2).

Tabla 2: Prueba t: Diferencias de las media en función del sexo.

\begin{tabular}{|l|r|r|}
\hline & \multicolumn{1}{|c|}{$\boldsymbol{H}$} & \multicolumn{1}{c|}{$\boldsymbol{M}$} \\
\hline Media & 16,10 & 15,85 \\
\hline Varianza & 3,51 & 3,46 \\
\hline Observaciones & 14,00 & 14,00 \\
\hline Varianza agrupada & 3,49 & \\
\hline Diferencia hipotética de las medias & 0,00 & \\
\hline Grados de libertad & 26,00 & \\
\hline Estadístico $\dagger$ & 0,34 & \\
\hline P(T<=†) una cola & 0,37 & \\
\hline Valor crítico de $\dagger$ (una cola) & 1,71 & \\
\hline P(T<=†) dos colas & 0,73 & \\
\hline Valor crítico de † (dos colas) & 2,06 & \\
\hline
\end{tabular}


Revista Tecné, Episteme y Didaxis: TED. Año 2014, Número Extraordinario. ISSN Impreso: 0121-3814, ISSN web: 2323-0126 Memorias, Sexto Congreso Internacional sobre Formación de Profesores de Ciencias. 08 al 10 de octubre de 2014, Bogotá

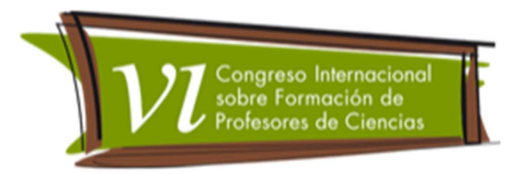

\section{Resultados I Medio A}

Del total de alumnos de la muestra, 30 corresponden al I medio A ( 15 mujeres y 15 hombres). Del total de subescalas del test, el que presentó las medias más altas frente a una actitud positiva y negativa es la Adopción de actitudes científicas (A) (media $=19,03, \mathrm{SD}=2,41$; media $=17,83, \mathrm{SD}=2,68$, respectivamente). Por consiguiente, la subescala que presentó las medias más bajas frente a una actitud positiva y negativa fue el Interés por la ciencia en el tiempo libre (L) (media $=13,03, \mathrm{SD}=4,34$; media $=14,00, \mathrm{SD}=4,53$, respectivamente).

Tabla 3: Estadística descriptiva I Medio A

\begin{tabular}{|c|c|c|c|c|c|c|c|}
\hline & $\begin{array}{l}\frac{\mathrm{\sigma}}{\mathrm{d}} \\
\sum^{\mathbb{0}}\end{array}$ & 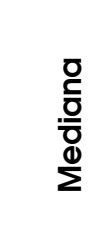 & $\begin{array}{l}\text { 융 } \\
\text { 일 }\end{array}$ & s & 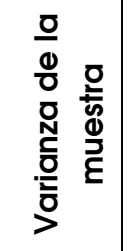 & $\begin{array}{l}\frac{\mathscr{D}}{D} \\
\stackrel{0}{0} \\
\frac{5}{5}\end{array}$ & 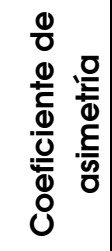 \\
\hline$S(+)$ & 18,50 & 18,00 & 17,00 & 2,24 & 5,02 & $-0,85$ & 0,19 \\
\hline$S(-)$ & 17,70 & 18,00 & 19,00 & 2,55 & 6,49 & $-0,23$ & $-0,04$ \\
\hline$N(+)$ & 17,80 & 18,00 & 21,00 & 3,25 & 10,58 & $-0,69$ & $-0,37$ \\
\hline$N(-)$ & 15,40 & 15,50 & 16,00 & 2,03 & 4,11 & 1,09 & $-0,24$ \\
\hline$I(+)$ & 17,77 & 18,00 & 18,00 & 3,07 & 9,43 & $-0,81$ & 0,00 \\
\hline$I(-)$ & 15,27 & 15,00 & 13,00 & 2,50 & 6,27 & 0,26 & 0,90 \\
\hline$A(+)$ & 19,03 & 20,00 & 21,00 & 2,41 & 5,83 & 0,28 & $-1,03$ \\
\hline$A(-)$ & 17,83 & 18,00 & 17,00 & 2,68 & 7,18 & 0,06 & $-0,36$ \\
\hline$E(+)$ & 14,77 & 14,50 & 14,00 & 3,65 & 13,29 & $-0,42$ & 0,37 \\
\hline$E(-)$ & 16,80 & 17,00 & 19,00 & 2,98 & 8,86 & 0,71 & 0,47 \\
\hline$L(+)$ & 13,03 & 13,00 & 13,00 & 4,34 & 18,86 & 1,42 & $-0,66$ \\
\hline$L(-)$ & 14,00 & 14,50 & 17,00 & 4,53 & 20,48 & $-0,92$ & 0,14 \\
\hline$C(+)$ & 13,90 & 13,00 & 13,00 & 3,86 & 14,92 & $-0,34$ & 0,60 \\
\hline
\end{tabular}


Revista Tecné, Episteme y Didaxis: TED. Año 2014, Número Extraordinario. ISSN Impreso: 0121-3814, ISSN web: 2323-0126 Memorias, Sexto Congreso Internacional sobre Formación de Profesores de Ciencias. 08 al 10 de octubre de 2014, Bogotá
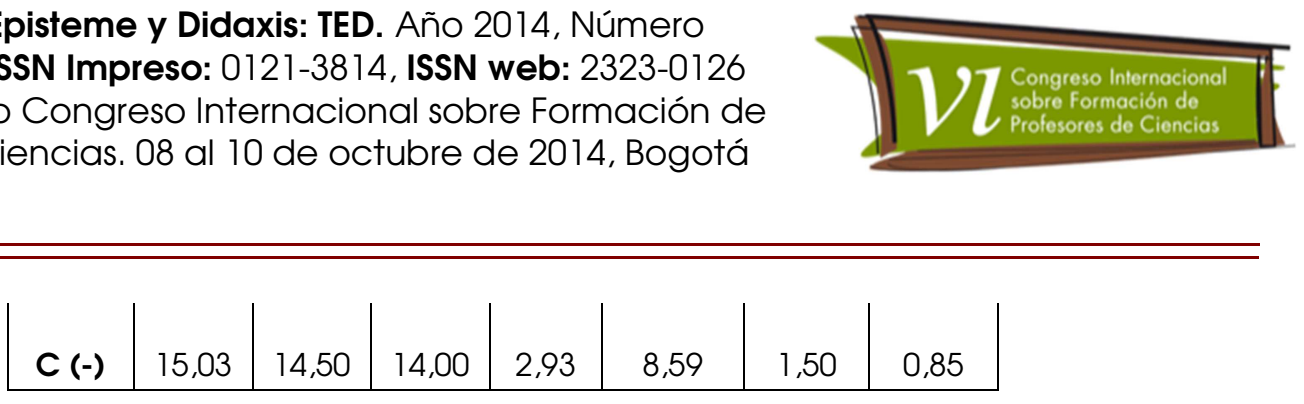

\section{$\underline{\text { Resultados I Medio B }}$}

Del total de alumnos de la muestra, 35 corresponden al I medio B ( 17 mujeres y 18 hombres). Del total de subescalas del test, el que presentó la media más alta frente a una actitud positiva es la Adopción de actitudes científicas (A) (media = 18,11 ; SD $=2,41$ ) y la subescala que presentó la media más alta frente a una actitud negativa fue las Implicaciones sociales de la ciencia (S) (media = 17,54; $S D=2,83$ ). Por consiguiente, la subescala que presentó la media más baja frente a una actitud positiva fue el Interés en carreras científicas $(C)$ (media = 12,23; SD = $3,56)$ y la subescala que presentó la media más baja frente a una actitud negativa fue el Interés por la ciencia en el tiempo libre $(L)$ (media = 14,00; SD = $3,42)$.

\begin{tabular}{|c|c|c|c|c|c|c|c|}
\hline & $\begin{array}{l}\frac{0}{0} \\
\frac{\mathbb{d}}{\Sigma}\end{array}$ & $\begin{array}{l}\text { o } \\
\stackrel{0}{0} \\
\frac{0}{0} \\
\stackrel{\Phi}{\Sigma}\end{array}$ & $\begin{array}{l}\text { 윰 } \\
\text { 일 }\end{array}$ & 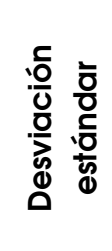 & 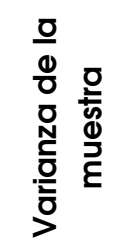 & $\begin{array}{l}\frac{n}{40} \\
\frac{0}{5} \\
0 \\
0\end{array}$ & 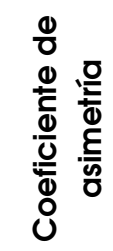 \\
\hline$S(+)$ & 17,49 & 18,00 & 20,00 & 2,55 & 6,49 & $-0,40$ & $-0,51$ \\
\hline$S(-)$ & 17,54 & 18,00 & 18,00 & 2,83 & 8,02 & 0,14 & $-0,19$ \\
\hline$N(+)$ & 17,23 & 17,00 & 19,00 & 3,52 & 12,36 & $-0,83$ & 0,10 \\
\hline$N(-)$ & 14,71 & 15,00 & 15,00 & 2,26 & 5,09 & 8,54 & 1,62 \\
\hline$I(+)$ & 17,57 & 18,00 & 14,00 & 3,66 & 13,37 & $-0,77$ & $-0,24$ \\
\hline$I(-)$ & 15,43 & 16,00 & 16,00 & 2,86 & 8,19 & $-0,12$ & $-0,52$ \\
\hline$A(+)$ & 18,11 & 19,00 & 20,00 & 2,41 & 5,81 & $-0,15$ & $-0,60$ \\
\hline$A(-)$ & 17,23 & 18,00 & 18,00 & 2,67 & 7,12 & 1,58 & $-0,77$ \\
\hline$E(+)$ & 14,57 & 15,00 & 18,00 & 4,16 & 17,31 & $-0,80$ & 0,15 \\
\hline$E(-)$ & 17,14 & 17,00 & 15,00 & 2,82 & 7,95 & $-0,53$ & $-0,15$ \\
\hline$L(+)$ & 13,89 & 14,00 & 16,00 & 4,62 & 21,34 & $-0,71$ & $-0,10$ \\
\hline
\end{tabular}


Revista Tecné, Episteme y Didaxis: TED. Año 2014, Número Extraordinario. ISSN Impreso: 0121-3814, ISSN web: 2323-0126 Memorias, Sexto Congreso Internacional sobre Formación de Profesores de Ciencias. 08 al 10 de octubre de 2014, Bogotá

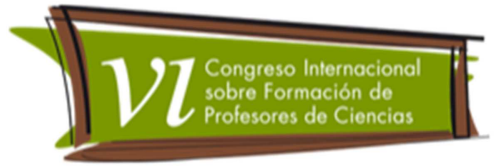

\begin{tabular}{|l|c|c|c|c|c|c|c|}
$\boldsymbol{L}(-)$ & 14,00 & 14,00 & 16,00 & 3,42 & 11,71 & 0,93 & $-0,36$ \\
\hline $\boldsymbol{C}(+)$ & 12,23 & 13,00 & 13,00 & 3,56 & 12,65 & $-0,55$ & $-0,15$ \\
\hline $\boldsymbol{C ( - )}$ & 14,14 & 14,00 & 11,00 & 3,54 & 12,54 & $-0,86$ & 0,14 \\
\hline
\end{tabular}

\section{Conclusiones}

De acuerdo a los resultados obtenidos en el pretest, los estudiantes de primer año medio del Colegio San Sebastián de Los Andes tienen una actitud positiva hacia una adopción de actitudes científicas por lo que se puede inferir que los estudiantes están dispuestos a adquirir conductas científicas en sus clases de ciencias. Sin embargo, presentan una actitud negativa frente a las implicaciones sociales de la ciencia, por lo que dentro del programa sería fundamental explicar las oportunidades y amenazas que las ciencias tienen en la sociedad actual.

De acuerdo a la prueba estadística t, no existen diferencias significativas entre hombres y mujeres frente a las actitudes hacia las ciencias.

\section{Referencias bibliográficas}

Acevedo, J. (2007). Consensos sobre la naturaleza de la ciencia: fundamentos de una investigación empírica. Revista Eureka sobre Enseñanza y Divulgación de las Ciencias, 4(1), pág. 42-66.

Escudero, T. (1995). La evaluación de las actitudes científicas. Didáctica de las ciencias experimentales, 2(4), pág. 33-41.

Fraser, B. (1981). Test of science related attitudes. Melbourne: Australian Council for Educational Research.

Gardner, P. (1975). Attitudes to science: A review. Studies in Science Education, 2, pág. 1-41.

Manassero, M. y Vásquez, A. (2001). Instrumentos y métodos para la evaluación de las actitudes relacionadas con la ciencia, la tecnología y la sociedad. Enseñanza de las ciencias, 20(1), pág. 15-27.

Navarro, M. y Forster, C. (2012). Nivel de alfabetización científica y actitudes hacia la ciencia en estudiantes de secundaria: comparaciones por sexo y nivel socioeconómico. Pensamiento Educativo. Revista de Investigación 
Revista Tecné, Episteme y Didaxis: TED. Año 2014, Número

Extraordinario. ISSN Impreso: 0121-3814, ISSN web: 2323-0126

Memorias, Sexto Congreso Internacional sobre Formación de

Profesores de Ciencias. 08 al 10 de octubre de 2014, Bogotá

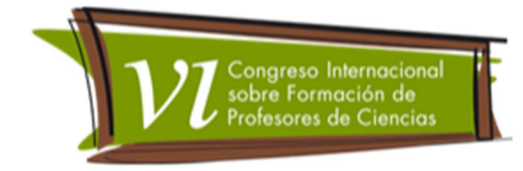

Educacional Latinoamericana, 49(1), pág. 1-17. Recuperado de http://pensamientoeducativo.uc.cl/index.php/pel/article/view/507/1082

OCDE (2006). PISA (2006). Marco de la evaluación. Conocimientos y habilidades en ciencias, matemáticas y lectura. Recuperado de www.oecd.org/dataoecd/59/39732471.pdf.

Vásquez et al. (2006). Actitudes del alumnado sobre ciencia, tecnología y sociedad evaluadas con un modelo de respuesta múltiple. Revista electrónica de investigación educativa, 8(2), 1-37. Recuperado de http://redie.uabc.mx/vol8no2/contenido-vasquez2.html 